\title{
A New Adaptive Parameterized Consistency Algorithm
}

\author{
Yunpeng $\mathrm{Wu}$ \\ College of Computer Science and Technology \\ Jilin University \\ Changchun, China \\ E-mail: 2412466779@qq.com
}

\author{
Yonggang Zhang * \\ College of Computer Science and Technology \\ Jilin University \\ Changchun, China \\ E-mail: zhangyg@jlu.edu.cn \\ * Corresponding Author
}

\author{
Sibo Zhang \\ College of Computer Science and Technology \\ Jilin University \\ Changchun, China \\ E-mail: 187097385@qq.com
}

\begin{abstract}
Nowadays all kinds of constraint solver support the same level of local consistency on all problems. The authors proposed a new adaptive parameterized consistency algorithm which adjusted the level of consistency depending on the depth of their supports in there domain. To this end, the authors gave the notion of the improved distance to the end of a value, which is the dynamic distance, and it reflected the current state of the assignment. If the size of the variable domain reached its given threshold or the ratio to the original size reached its given threshold, the constraint solving process used the stronger consistency technique to propagate the assigned value. The faster deletion of invalid value, the easier cause backtracking. It is more likely to find the problem as soon as possible. Finally the authors carry on the experiments on some benchmark instances, the results showed that the new algorithm can outperform original parameterized consistency algorithm on many problems and is a promising method.
\end{abstract}

Keywords-Constraint Satisfaction Problem; Constraint Propagation; Adaptive; Backtracking; Parameterized Consistency

\section{INTRODUCTION}

Constraint programming (CP) is a powerful paradigm for solving combinatorial search problems that draws on a wide range of techniques from artificial intelligence, computer science, databases, programming languages, and operations research. Constraint programming is currently applied with success to many domains, such as scheduling, planning, vehicle routing, configuration, networks, and bioinformatics [1]. Constraint satisfaction problems (CSPs) are the central problems in $\mathrm{CP}$, which are mathematical problems defined as a set of objects whose state must satisfy a number of constraints or limitations. CSPs represent the entities in a problem as a homogeneous collection of finite constraints over variables, which were solved by constraint satisfaction methods. CSPs are the subject of intense research in both artificial intelligence and operations research, since the regularity in their formulation provides a common basis to analyze and solve problems of many unrelated families. CSPs often exhibit high complexity, requiring a combination of heuristics and combinatorial search methods to be solved in a reasonable time.

Backtracking and constraint propagation are two main and basic constraint solving methods [1]. Arc consistency is the oldest and most famous way of constraint propagation. It can be used to prune infeasible values in the preprocessing and backtracking phase. Arc consistency is the standard level of consistency supported in constraint solvers. In addition, several other local consistencies stronger than arc consistency have been proposed, such as max restricted path consistency[3] or singleton arc consistency[4] and their variants[6,7,8]. Unfortunately, these new consistency techniques do not play the important role in current constraint solvers because of the high computational cost of maintaining them during search.

Stergiou[9] found that choosing the right level of local consistency for solving a problem required making the trade-off between the ability of removing inconsistent values and the cost of the algorithm. And he also suggested take advantage of the power of strong consistencies to reduce the search space while avoiding the high maintain ing cost in the whole network. His paper presented a heuristic approach based on the monitoring of propagation events to dynamically adapt the level of local consistency (arc consistency or max restricted path consistency) to individual constraints. This pruned more values than arc consistency and less than max restricted path consistency, but it was more efficient than the arc consistency and max restricted path consistency.

Recently, Balafrej[10] proposed an original 
parameterized consistency method. The paper introduced the notion of stability of values for arc consistency, a notion based on the depth of their supports in their do main. The technique allowed defining levels of local consistency of increasing strength between arc consistency and a given strong local consistency. It has instantiated the generic parameterized consistency approach to $\max$ restricted path consistency. The experiments showed that the parameterized consistency technique is viable and adapting the level of local consistency during search using the parameterized consistency is a promising approach that can outperform both MAC and a strong local consistency on many problems.

In this paper the authors define the notion of the improved distance to end of a value. It was the improved notion of the notion in the Balafrej's paper. The authors have observe that the distance to end of a value keeping the same value during the whole constraint solving process, it can't reflect the accurate information guiding the weak or strong consistencies enforcement. The improved distance to end of a value and corresponding thresholds resulted the new p-maxRPC ${ }^{\text {imp }}$ algorithm could enforce the strong consistency when needed, so it could increase the constant solving efficiency. The experiments results showed that the new proposed method was more efficient than the original algorithm.

The remainder of this paper is structured as followes. In Section 2, the authors introduced the background of the CSP and constraint propagation. In section 3, the authors discussed the parameterized consistency notion and the algorithm. In Section 4, the authors define the notion of the improved distance to end of a value and presented the $p$ maxRPC ${ }^{i m p}$ algorithm. In Section 5, the authors gave the results and analysis of the experiments. In Section 6, the authors brief summarized the whole paper and pointed out the future work.

\section{BACKGROUND}

A constraint network $\mathrm{N}$ is defined as set of $\mathrm{n}$ variables $\mathrm{X}=\left\{\mathrm{x}_{1}, \mathrm{x}_{2}, \cdots, \mathrm{x}_{\mathrm{n}}\right\}, \quad \mathrm{a}$ set of ordered domain $\mathrm{D}=\left\{\mathrm{D}\left(\mathrm{x}_{1}\right), \mathrm{D}\left(\mathrm{x}_{2}\right), \cdots, \mathrm{D}\left(\mathrm{x}_{\mathrm{n}}\right)\right\}$, and a set of $\mathrm{e}$ constraints $\mathrm{C}=\left\{\mathrm{c}_{1}, \mathrm{c}_{2}, \cdots, \mathrm{c}_{\mathrm{e}}\right\}$. Each constraint $\mathrm{c}_{\mathrm{k}}$ is defined by a pair( $\left.\operatorname{var}\left(c_{k}\right), r\left(c_{k}\right)\right)$, where $\operatorname{var}\left(c_{k}\right)$ is an ordered subset of $X$, and $r\left(c_{k}\right)$ is a set of combinations of values satisfying $c_{k}$.In the following, the authors restrict binary CSP.A binary constraint $c$ between $x_{i}$ and $x_{j}$ will be denote by $c_{i j}$, and $\mathrm{C}\left(\mathrm{x}_{\mathrm{i}}\right)$ denote the set of variables $\mathrm{x}_{\mathrm{j}}$ involved in a constraint with $x_{i}$.

A value $v_{j} \in D\left(x_{j}\right)$ is called an arc consistent support(AC support)for $v_{i} \in D\left(x_{i}\right)$ on $c_{i j}$ if $\left(v_{i}, v_{j}\right) \in r\left(c_{i j}\right)$.A value $\mathrm{v}_{\mathrm{i}} \in \mathrm{D}\left(\mathrm{x}_{\mathrm{i}}\right)$ is arc consistent(AC) if and only if for all $\mathrm{x}_{\mathrm{j}} \in \mathrm{C}\left(\mathrm{x}_{\mathrm{i}}\right), \mathrm{v}_{\mathrm{i}}$ has an $\mathrm{AC}$ support $\mathrm{v}_{\mathrm{j}} \in \mathrm{D}\left(\mathrm{x}_{\mathrm{j}}\right)$. A domain $\mathrm{D}\left(\mathrm{x}_{\mathrm{i}}\right) \mathrm{us}$ arc consistent if it is non empty and all values in $\mathrm{D}\left(\mathrm{x}_{\mathrm{i}}\right)$ are arc consistent. A network is arc consistent if all domains in $\mathrm{D}$ are arc consistent. If enforcing arc consistency on a network $\mathrm{N}$ leads to a domain wipe out, the authors say the $\mathrm{N}$ is arc inconsistent. A tuple $\left(\mathrm{v}_{\mathrm{i}}, \mathrm{v}_{\mathrm{j}}\right) \in \mathrm{D}\left(\mathrm{x}_{\mathrm{i}}\right) \times \mathrm{D}\left(\mathrm{x}_{\mathrm{j}}\right)$ is path consistent $(\mathrm{PC})$ if and only if for any third variable $\mathrm{x}_{\mathrm{k}}$ there exists a value $v_{k} \in D\left(x_{k}\right)$ such that $v_{k}$ is an AC support for both $v_{i}$ and $v_{j}$, and $v_{k}$ is called witness for the path consistency of $\left(\mathrm{v}_{\mathrm{i}}, \mathrm{v}_{\mathrm{j}}\right)$.
A value $v_{j} \in D\left(x_{j}\right)$ is a max restricted path consistent (maxRPC) support for $\mathrm{v}_{\mathrm{i}} \in \mathrm{D}\left(\mathrm{x}_{\mathrm{i}}\right)$ on $\mathrm{c}_{\mathrm{ij}}$ if and only if it is an AC support and the tuple $\left(v_{i}, v_{j}\right)$ is path consistent. A value $v_{i} \in D\left(x_{i}\right)$ is max restricted path consistent of a constraint $c_{i}$ if and only if there is oo a $v_{j} \in D\left(x_{j}\right)$ ) maxRPC support for $v_{i}$ on $c_{i j}$. A value $v_{i} \in D\left(x_{i}\right)$ is max restricted path consistent if and only if for all $x_{j} \in C\left(x_{i}\right) v_{i}$ has a maxRPC support $\mathrm{v}_{\mathrm{j}} \in \mathrm{D}\left(\mathrm{x}_{\mathrm{j}}\right)$ on $\mathrm{c}_{\mathrm{ij}}$. A domain $\mathrm{D}\left(\mathrm{x}_{\mathrm{i}}\right)$ is maxRPC if it is non empty and all values in $\mathrm{D}\left(\mathrm{x}_{\mathrm{i}}\right)$ are maxRPC. A constraint network $\mathrm{N}$ is maxRPC if all domains in $\mathrm{D}$ are maxRPC.

The problem of finding the solution of a constraint network is called constrain solving, and it is NP-complete. The basic solving method is backtrack search combined with the some level of consistency.

\section{PARAMETERIZED CONSISTENCY}

Parameterized consistency was based on the concept of stability of values. Balafrej introduce the notion of the distance to end of a values in a domain. This captured how far a value was from the last in its domain. In the following, the definition and the theorem came from[10].

Definition 1.(Distance to end of a value).The distance to end of a value $\mathrm{v}_{\mathrm{i}} \in \mathrm{D}\left(\mathrm{x}_{\mathrm{i}}\right)$ is the ratio

$$
\operatorname{Dis}\left(x_{i}, v_{i}\right)=\left(\left|D_{0}\left(x_{i}\right)\right|-\operatorname{rank}\left(v_{i}, D_{0}\left(x_{i}\right)\right)\right) /\left|D_{0}\left(x_{i}\right)\right|
$$

where $\mathrm{D}_{0}\left(\mathrm{x}_{\mathrm{i}}\right)$ is the initial domain of $\mathrm{x}_{\mathrm{i}}$.

Definition 2.(p-stability for $A C$ ) $A$ value $v_{i} \in D\left(x_{i}\right)$ is $\mathrm{p}$-stable for $A C$ on $c_{i j}$ iff $v_{i}$ has an $A C$ support $v_{j} \in D\left(x_{j}\right)$ on $c_{i j}$ such that Dis $(x j, v j) \geqslant$ p.A value $v_{i} \in D\left(x_{i}\right)$ is $p$-stable for AC iff for all $x_{j} \in C\left(x_{i}\right), v_{i}$ is p-stable for $A C$ on $c_{i j}$.

Theorem 1. Let LC be a local consistency stronger than AC for which the LC consistency of a value on a constraint is defined. Let $\mathrm{p}_{1}$ and $\mathrm{p}_{2}$ be two parameters in [0..1]. If $\mathrm{p}_{1}<\mathrm{p}_{2}$ then $\mathrm{AC}<$ constraint-based $\mathrm{p}_{1}-\mathrm{LC}<$ constraint-based $\mathrm{p}_{2}-\mathrm{LC}<\mathrm{LC}$.

Theorem 2. Let $\mathrm{LC}$ be a local consistency stronger than AC. Let $\mathrm{p}_{1}$ and $\mathrm{p}_{2}$ be two parameters in [0..1]. If $\mathrm{p}_{1}<$ p2 then $\mathrm{AC}<$ value-based $\mathrm{p}_{1}-\mathrm{LC}<$ value-basedp2-LC $<$ LC.

Definition3. (p-maxRPC). A value and a network are $\mathrm{p}$-maxRPC if and only if they are constraint-based $\mathrm{p}$ maxRPC.

\section{P-MAXRPC ${ }^{\mathrm{IMP}}$ ALGORITHM}

Definition 4. (Improved Distance to end of a value).The Improved distance to end of a value $\mathrm{v}_{\mathrm{i}} \in \mathrm{D}\left(\mathrm{x}_{\mathrm{i}}\right)$ is the ratio

$$
\operatorname{Dis}\left(x_{i}, v_{i}\right)=\left(\left|D_{c u}\left(x_{i}\right)\right|-\operatorname{rank}\left(v_{i}, D_{c u}\left(x_{i}\right)\right)\right) /\left|D_{c u}\left(x_{i}\right)\right|
$$

Where, $\mathrm{D}_{\mathrm{cu}}\left(\mathrm{x}_{\mathrm{i}}\right)$ is the current domain of $\mathrm{x}_{\mathrm{i}}$.

During the process of solving the variable universe, the authors have the priority to implement the principle of priority in the process of the current domain size to reach a certain limit. According to the experimental data summarized the threshold set to a and $b$. That $\left|D_{c u}\left(x_{j}\right)\right| \leqslant a$ or $\left|D_{c u}\left(x_{i}\right) /\right| D_{o}\left(x_{i}\right) \mid \leqslant b$, the $b$ is measure the current on the domain size of the standard, the current on the domain size and initial on the domain size, the ratio is less than beta determine when the domain of the variable value up to standard, does not have to perform comparis on $\left(\mathrm{x}_{\mathrm{i}}, \mathrm{v}_{\mathrm{i}}\right)$ with $\mathrm{P}$, direct use of strong consistency checking algorithm, thus ensuring more backtracking to the premise with 
minimal overhead were judged. Therefore, it is easier to produce a dead node so as to lead to backtracking and improve the efficiency of solving. The followed were the pseudo code

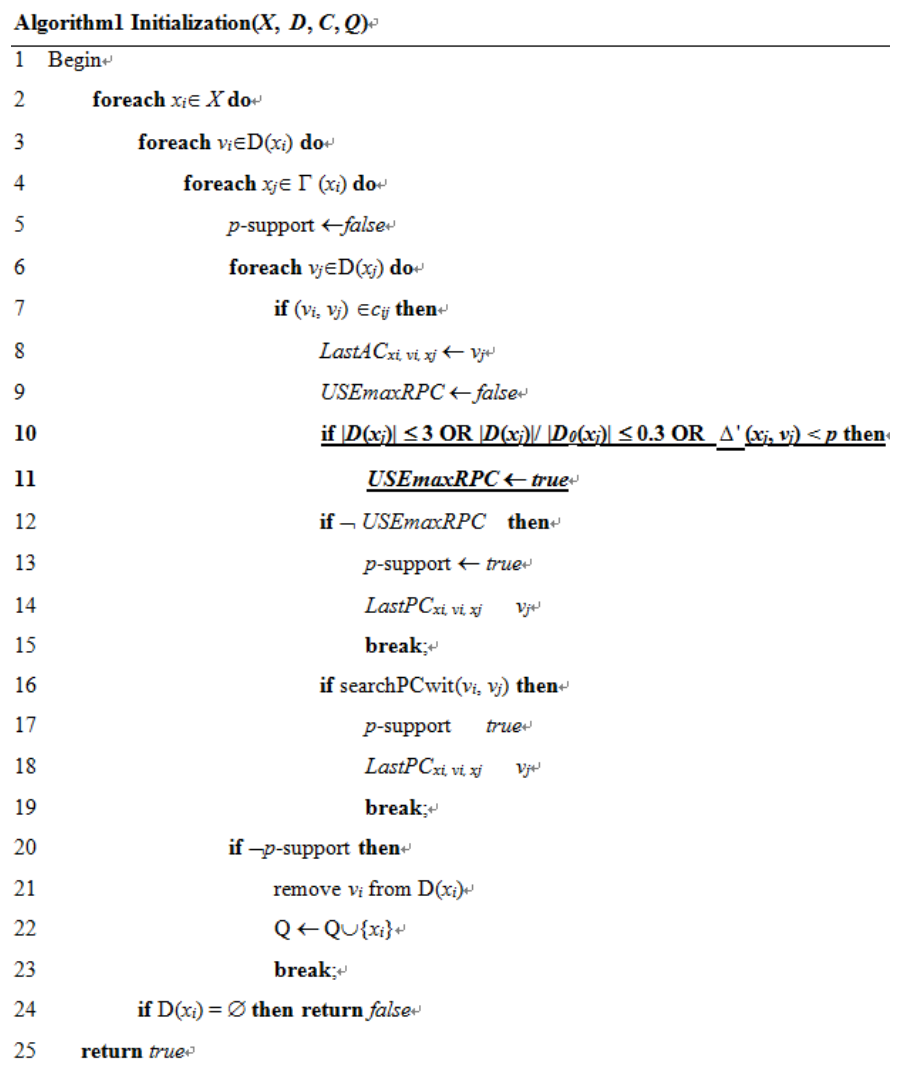

Figure 1. Algorithm1 Initialization

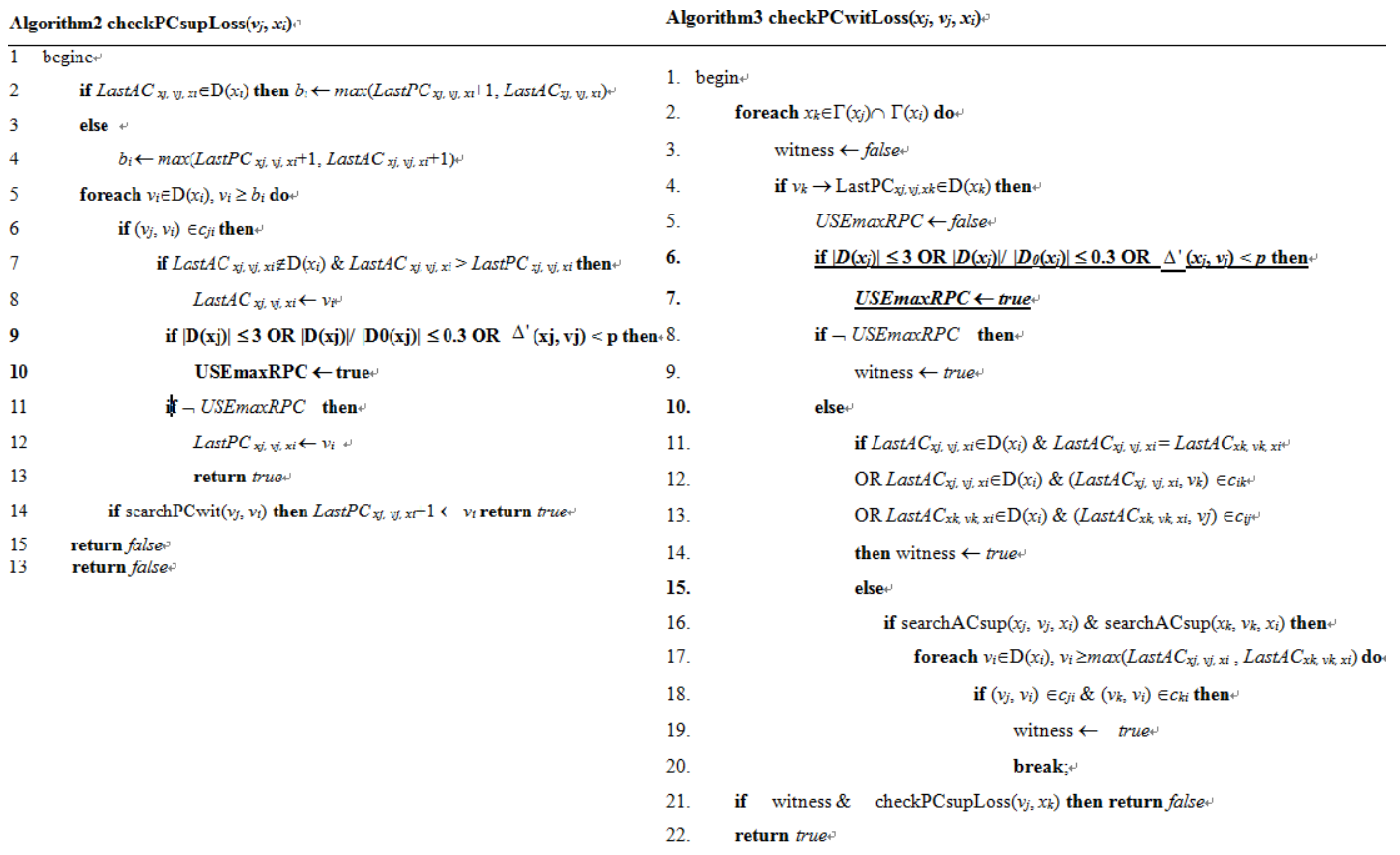

Figure 2. Algorithm 2 checkPCsupLoss and Algorithm 3 checkPC witLoss

The line 9 of the algorithm 1 initialization (X, D, C, Q) (Fig. 1), line 9 of the algorithm 2checkPCsupLoss $\left(v_{j}\right.$, $\left.\mathrm{x}_{\mathrm{i}}\right)$ and the line $t$ of the algorith $\mathrm{m} 3$ checkPCwitLoss ( $\mathrm{x}_{\mathrm{j}}$, $\left.\mathrm{v}_{\mathrm{j}}, \mathrm{x}_{\mathrm{i}}\right)$, the authors add the the $\left|\mathrm{D}\left(\mathrm{x}_{\mathrm{j}}\right)\right| \leqslant 3$ and $\left|\mathrm{D}\left(\mathrm{x}_{\mathrm{j}}\right)\right|$ $\left|\mathrm{D}_{0} \quad\left(\mathrm{x}_{\mathrm{j}}\right)\right| \leqslant 0.3$ judgment, namely, to determine the current domain size, if on a smaller domain, use the 
delete value ability strongly consistent algorithm, so more easily produced dead node as soon as possible so as to lead to backtracking, improve the efficiency of the algorithm.

\section{EXPERIMENTS}

In this section, the authors will show the experimental evaluation of the proposed algorithm. All the problem instances come from the website (http://www.cril.univartois.fr/ lecoutre/benchmarks.html\#) maintained by Lecoutre. All algorithms have been implemented in $\mathrm{C}++$ and embedded into the constraint solving platform designed by ourselves. In this platform, the authors use $\mathrm{dom} / \mathrm{deg}$ variable ordering because it is easy to implement and very efficient. The experiments were carried out on a DELL Intel i7-3770 $3.40 \mathrm{GHz}$ CPU/8GB RAM with Windows 7.0/Visual C++6.0.

T ABLE I. PERFORMANCE (CPU TIME, NODES AND CONSTRAINT CHECKS) OF MAXRPC, VARIABLE-BASED AP-MAXRPC(APX-MANRPC), CONSTRAINTBASED AP-MAXRPC(APC-MAXRPC) AND THE CORRESPONDING IMPROVED VERSION ON QWH INSTANCES

\begin{tabular}{|c|c|c|c|c|c|}
\hline \multirow{2}{*}{$\begin{array}{c}\text { Instances } \\
\quad \text { (sat) }\end{array}$} & \multirow{2}{*}{ Measure } & \multirow{2}{*}{$\operatorname{maxRPC}$} & p-maxRPC & apc-maxRPC & $\operatorname{apx}-\max R P C$ \\
\hline & & & $p-m a x R P C^{\text {imp }}$ & apc-maxRPC ${ }^{\text {imp }}$ & $\operatorname{apx}-m a x R^{\prime m p}$ \\
\hline \multirow{2}{*}{ qwh-15-106-0_ext } & $\begin{array}{l}\text { cpu(s) } \\
\text { \#ccks } \\
\text { \#nodes }\end{array}$ & \multirow{2}{*}{$\begin{array}{c}307.551 \\
4348296 \\
1856\end{array}$} & Time-out & $\begin{array}{c}479.477 \\
2857255 \\
3893\end{array}$ & $\begin{array}{c}302.539 \\
3945798 \\
1856\end{array}$ \\
\hline & $\begin{array}{l}\text { cpu(s) } \\
\text { \#ccks } \\
\text { \#nodes }\end{array}$ & & $\frac{302.173}{4299125}$ & $\begin{array}{c}310.1 \\
4296012 \\
1879\end{array}$ & $\begin{array}{c}275.95 \\
4358267 \\
1856\end{array}$ \\
\hline \multirow{2}{*}{ qwh-15-106-2_ext } & $\begin{array}{l}\text { cpu(s) } \\
\text { \#ccks } \\
\text { \#nodes }\end{array}$ & \multirow{2}{*}{$\begin{array}{c}522.875 \\
7976700 \\
2801\end{array}$} & $\begin{array}{c}3585.64 \\
28010711 \\
24135\end{array}$ & $\begin{array}{c}1179.71 \\
5660938 \\
9287\end{array}$ & $\begin{array}{c}524.15 \\
7091034 \\
2801\end{array}$ \\
\hline & $\begin{array}{l}\text { cpu(s) } \\
\text { \#ccks } \\
\text { \#nodes }\end{array}$ & & $\begin{array}{c}566.563 \\
8136404 \\
2990\end{array}$ & $\begin{array}{c}565.207 \\
8133621 \\
2990\end{array}$ & $\begin{array}{c}\frac{516.075}{8030984} \\
2801\end{array}$ \\
\hline \multirow{2}{*}{ qwh-15-106-3_ext } & $\begin{array}{l}\text { cpu(s) } \\
\text { \#ccks } \\
\text { \#nodes }\end{array}$ & \multirow{2}{*}{$\begin{array}{c}26.949 \\
480361 \\
230\end{array}$} & $\begin{array}{c}56.097 \\
518713 \\
471\end{array}$ & $\begin{array}{c}34.947 \\
124863 \\
359\end{array}$ & $\begin{array}{c}23.508 \\
367257 \\
230\end{array}$ \\
\hline & $\begin{array}{l}\text { cpu(s) } \\
\text { \#ccks } \\
\text { \#nodes }\end{array}$ & & $\begin{array}{c}73.039 \\
409856 \\
230\end{array}$ & $\begin{array}{c}27.368 \\
409847 \\
230\end{array}$ & $\begin{array}{c}\frac{22.564}{446423} \\
230\end{array}$ \\
\hline \multirow{2}{*}{ qwh-15-106-4_ext } & $\begin{array}{l}\text { cpu(s) } \\
\text { \#ccks } \\
\text { \#nodes }\end{array}$ & \multirow{2}{*}{$\begin{array}{c}1.859 \\
118680 \\
104\end{array}$} & $\begin{array}{c}1.675 \\
60896 \\
114\end{array}$ & $\begin{array}{c}1.822 \\
23392 \\
138\end{array}$ & $\begin{array}{c}1.652 \\
97603 \\
104\end{array}$ \\
\hline & $\begin{array}{l}\text { cpu(s) } \\
\text { \#ccks } \\
\text { \#nodes }\end{array}$ & & $\begin{array}{c}\frac{1.571}{71664} \\
104\end{array}$ & $\begin{array}{c}1.573 \\
71920 \\
104\end{array}$ & $\begin{array}{c}1.685 \\
107256 \\
104\end{array}$ \\
\hline \multirow{2}{*}{ qwh-15-106-6_ext } & $\begin{array}{l}\text { cpu(s) } \\
\text { \#ccks } \\
\text { \#nodes }\end{array}$ & \multirow{2}{*}{$\begin{array}{c}69.254 \\
1115837 \\
421\end{array}$} & $\begin{array}{c}179.123 \\
2538845 \\
1517\end{array}$ & $\begin{array}{c}101.846 \\
571642 \\
690\end{array}$ & $\begin{array}{c}62.659 \\
952177 \\
422\end{array}$ \\
\hline & $\begin{array}{l}\text { cpu(s) } \\
\text { \#ccks } \\
\text { \#nodes }\end{array}$ & & $\begin{array}{c}\frac{61.54}{038729} \\
421\end{array}$ & $\begin{array}{c}66.831 \\
1039289 \\
421\end{array}$ & $\begin{array}{c}66.828 \\
1092199 \\
421\end{array}$ \\
\hline \multirow{2}{*}{ qwh-15-106-7_ext } & $\begin{array}{l}\text { cpu(s) } \\
\text { \#ccks } \\
\text { \#nodes }\end{array}$ & \multirow{2}{*}{$\begin{array}{c}536.533 \\
6693875 \\
2896\end{array}$} & Time-out & $\begin{array}{c}1256.66 \\
7291572 \\
10069\end{array}$ & $\begin{array}{c}543.576 \\
6156600 \\
3003\end{array}$ \\
\hline & $\begin{array}{l}\text { cpu(s) } \\
\text { \#ccks } \\
\text { \#nodes }\end{array}$ & & $\begin{array}{c}\frac{533.014}{6549992} \\
2874\end{array}$ & $\begin{array}{c}533.657 \\
6553730 \\
2874\end{array}$ & $\begin{array}{c}534.168 \\
6672117 \\
2900\end{array}$ \\
\hline
\end{tabular}


All the instances in TABLE I are belong to the SAT (satisfaction type), where each instance including 100 variables and 900 constraints. The experiments showed that the improved algorithms have advantage both in solving time and visited nodes. Compared with the classical maxRPC algorithm, the adaptive version of maxRPC algorithms were not as good as the classical maxRPC on the most instances. It showed that the adaptive version algorithm increase the computational cost though they decreased the strong consistency cost. The authors could observe the data about p-maxRPC, apc-maxRPC, apx-maxRPC algorithm, and could find that apx-maxRPC was the best one.It was because that this algorithm adjusted threshold $\mathrm{p}$ using variable-based method, the value of $\mathrm{p}$ became bigger and bigger with the domain reduced, thus it enforced strong consistency more frequently and got the solution more quickly. The improved p-maxRPC, apc-maxRPC, apx-maxRPC had stable advantage both on the CPU time and number of the visited node. The execution time is generally less than the original algorithms, and the number of nodes is the same as those of the original algorithms.

In the original three parameters of the algorithm can be seen based on the adaptive variable is a absolute advantage, the reasons above have given analysis. By comparison, the new three parameter apx-maxRPC ${ }^{\text {imp }}$ algorithm is more efficient than the algorithm based on variable in the same optimization algorithm. Although the overall execution time is less than before optimization, most of the algorithms and the visit to the number of nodes is minimized, but apx-maxRPC ${ }^{i m p}$ in based on the method of adaptive variable optimization is not perfect. This is mainly because the idea is to optimize the value of the idea is to update the value of the end of the distance, while the variable is also the variable adaptive and it is worth to cut and continue to change. Based on the variation of the threshold parameters of the variable is by all variables where the median constraint deletion caused, but terminal distance changes indeed within the limited scope changes, this range is the initial domain size. Therefore, change from the end of distance speed to change the parameters of the threshold $p$ value range. The implementation of the algorithm is less than the value of the $p$ value of the implementation of the strong compatibility check. In front of the initial domain value because of distance is greater than the threshold; it is not strong compatibility check. However, due to the rapid increase of $\mathrm{P}$ value, it is not required to perform the strong compatibility check of the value of the end of the scale is less than $P$, which makes a partial value of the early implementation of the strong compatibility check. As a result, the strong consistency checks led to the increase in the implementation of the time.

\section{CONCLUSIONS}

State-of-the-art constraint solvers uniformly maintain the same level of local consistency on all the instances.
The authors proposed a new parameterized local consistency based on the notion of the improved distance to the end of a value, which is the dynamic distance, and it reflected the current state of the assignment. If the size of the variable do main reached its given threshold or the ratio to the original size reached its given threshold, the constraint solving process used the stronger consistency technique to propagate the assigned value. The faster deletion of invalid value, the easier cause backtracking. It is more likely to find the problem as soon as possible. Finally the authors carry on the experiments on some benchmark instances, the results showed that the new algorithm can outperform original parameterized consistency algorithm on many problems and is a promising method.

\section{ACKNOWLEDGMENT}

The authors of this paper express sincere gratitude to all the anonymous reviewers for their hard work. This work was supported in part by NSFC under Grant No. 61170314, No. 61373052.

\section{REFERENCES}

[1] F.Rossi, P.van Beek, T.Walsh, Handbook of Constraint Programming.Elsevier, 2006

[2] T. Balafoutis, A.Paparrizou, K.Stergiou, T.Walsh, "New algorithms for max restricted path consistency," Constraints, volume16, pp.372-406,August 2011, doi: 10.1007/s10601-0119110-y.

[3] C.Bessiere, J.C.Regin, R.H.C.Yap, Y.Zhang, "An optimal coarse-grained arc consistency algorithm," Artificial Intelligence, volume 165, pp.165-185,Feb.2005, doi: 10.1016/j.artint.2005.02.004.

[4] C.Bessiere, K.Stergiou, T.Walsh, "Domain filtering consistencies for non-binary constraints,". Artificial Intelligence, volume172,pp.800-822,Feb.2008, doi: 10.1016/j.artint. 2007. 10. 016 .

[5] R.Debruyne, C.Bessiere, "Domain Filtering consistencies", Journal of Artificial Intelligence Research.volume 14, pp.205230,Oct.2001,doi: 10.1613/jair.834

[6] K.Stergiou, "Heuristics for dynamically adapting propagation in constraint satisfaction problems," AI Commun,volume22, pp.125-141,August 2009, doi: 10.3233/AIC-2009-0450

[7] I.Katriel, P. Van Hentenryck, "Randomized filtering algorithms,'Technical ReportCS-06-09, Brown University „June 2006.

[8] M.Sellmann, “Approximated consistency for knapsack constraints," Proc. International Conference on Principles and Practice of Constraint Programming 2003(CP03), Springer Press, 2003, Oct.2003,pp.679-693,doi: 10.1007/978-3-540-45193-8_46.

[9] A. Balafrej, C. Bessiere, R. Coletta, E.-H. Bouyakhf, "Adaptive Parameterized Consistency,". Proc. International Conference on Principles and Practice of Constraint Programming (CP13), Springer Press, 2013, Sept.2013,pp.143-158, doi: 10.1007/978-3642-40627-0_14.

[10] R. J. Woodward, A. Schneider, B.Y. Choueiry, C. Bessiere, "Adaptive Parameterized Consistency for Non-binary CSPs by Counting Supports," Proc. International Conference on Principles and Practice of Constraint Programming (CP14), Springer Press, 2014, Sept .2014,pp.755-764, doi: 10.1007/978-3319-10428-7_54. 